\title{
Enfermedades afectivas y actividad solar. Seguimiento a 16 años
}

\author{
FERNANDO IVANOVIC-ZUVIC ${ }^{1}$, RODRIGO DE LA VEGA ${ }^{2}$, \\ NEVENKA IVANOVIC-ZUVIC ${ }^{2}$, EDUARDO CORREA $^{1}$
}

Clínica Psiquiátrica

Universitaria, Facultad de Medicina, Universidad de Chile. ${ }^{2}$ Círculo de Santiago, Centro de Estudios Científicos Santiago de Chile.

Recibido el 7 de agosto de 2009, aceptado el 12 de mayo de 2010.

Correspondencia a: Fernando Ivanovic-Zuvic Callao 2970 of. 604. Las Condes, Santiago, Chile Fono: $56-2-2324672$ E-mail: ferlore@vtr.net

\section{Association between hospital admissions due to affective disorders and solar activity. Analysis of 16 years}

Background: Exposure to solar activity may be associated with incidence of depressive and manic disorders. Aim: To assess the link between solar activity and appearance of affective disorders. Material and Methods: We examined $1862 \mathrm{cli}$ nical records of a psychiatric clinic located in Santiago, Chile. Patients with major depression and manic disorders were included in the study, only when they were admitted at the clinic for the first time. Solar activity was calculated using the Wolf number, which is given by the formula $R=K(10 g+f)$, where " $g$ " stands for the groups of sunspots and " $f$ " is the total number of sunspots. We examined the correlation between annual incidence of hospital admissions and average Wolf numbers for the period 1990-2005, which corresponds to approximately one and half solar cycles of 16 years. Results: A total of 450 medical records corresponding to 299 patients (199 women) with depressive symptoms and 151 patients (73 women) with mania, were analyzed. There was a higher number of admissions for depression during the years with lower solar activity. Admissions due to mania tended to increase in the years with high solar activity. There was a negative correlation between the number of hospital admissions due to depression and solar activity (Spearman $r=-0.812, p<0.01$ ). The association between the latter parameter and admissions due to mania did not reach statistical significance. Conclusions: There is a significant negative association between the rate of hospital admissions due to depressive disorders and solar activity.

(Rev Med Chile 2010; 138: 694-700).

Key words: Bipolar disorder; Depression; Solar activity.
$\mathrm{E}$ 1 Sol influye sobre la Tierra de las más diversas maneras, desde la presencia de formas de vida hasta cambios de temperatura y estaciones. La actividad solar es expresada por la luz emitida, pero también por fuentes de intensa actividad energética tales como los rayos $\mathrm{X}$ y ultravioletas, así como otras varias formas de energía, las cuales constituyen el espectro solar.

Diversos trastornos mentales han sido vinculados con el influjo de los astros sobre el ser humano. Los más estudiados han sido las enfermedades anímicas, especialmente los trastornos afectivos estacionales (SAD, sigla en inglés para "seasonal affective disorders") ${ }^{1}$. Se ha señalado que los portadores de SAD de invierno poseen como características clínicas síntomas depresivos atípicos $^{1-5}$, mientras que los SAD de verano cursan con síntomas típicamente endógenos ${ }^{2}$.

Se menciona que la mayor incidencia de síntomas en el invierno en sujetos depresivos no se correlacionaría con la nubosidad, lluvias o presión atmosférica, aunque sí con la cantidad de luz solar y duración del día, lo que avalaría la influencia de la luz sobre estas depresiones ${ }^{6}$. De este modo, 
algunos autores destacan el rol que juega el fotoperíodo en la patogénesis de los SAD en invierno como el factor más relevante comparado con otras variables ambientales ${ }^{7}$.

Es conocido el efecto de la fototerapia como tratamiento efectivo para los cuadros depresivos, tanto en SAD como en aquellos que no siguen este patrón $\mathrm{n}^{8-10}$. Se ha mencionado que la melatonina, hormona secretada por la glándula pineal especialmente durante la noche, sería un factor concurrente. Su secreción está controlada por el núcleo supraquiasmático que recibe estímulos fóticos desde la retina. La acción de la melatonina puede ser suprimida por la luz brillante artificial modificando su patrón de fase y de este modo influyendo sobre el estado anímico que se vincula a estos marcapasos ${ }^{11,12}$. La magnitud de su supresión depende de la cantidad de luz blanca brillante aplicada medida a través de unidades $l u x^{13}$, efecto que ocurre tanto en sujetos deprimidos como en adultos normales, alterando los marcapasos circadianos y el patrón del sueño ${ }^{14-21}$, influyendo en los niveles de melatonina plasmática, de prolactina y cortisol $^{22,23}$.

La actividad energética del sol podría ser otra variable correlacionada con la aparición de enfermedades afectivas. Ya en 1610 Galileo Galilei observó manchas solares desde un telescopio, aunque astrónomos chinos las reconocieron y registraron al menos un milenio antes, aunque no de manera metódica y científica. Estas manchas corresponden a poros que varían en número, dimensión y profundidad a lo largo del ciclo solar y emiten energía en la forma de actividad magnética parcialmente visible.

Estas manchas poseen una variación periódica a lo largo del tiempo. Fue Heinrich Schrabe quien en 1843 descubrió que la repetición de la frecuencia máxima de las manchas tiene lugar cada 11 años aproximadamente. Durante este período, las manchas presentan un comportamiento regular con un máximo alcanzando unas 25-30 al día y un mínimo en que no se observan por varios días ${ }^{24}$.

Desde 1849 se vienen efectuando mediciones de estas manchas, las que fueron sistematizadas por Wolf, quien denominó a esta alza y disminución de ellas en períodos regulares de 11 años, como un "ciclo solar", que se repite en forma constante y calculó un índice que refleja la emisión de energía, el denominado "número de Wolf".

Las manchas solares están relacionadas directa- mente con la emisión de energía solar o actividad solar que se refleja en el espectro de radiaciones que va desde los rayos gama, de mayor energía, seguido de los rayos ultravioletas y los infrarrojos de menor energía. En la medida que aumentan las manchas solares, aumenta la emisión de energía bajo esta forma de espectro de radiaciones, donde la luz visible es sólo una parte pequeña, delimitada por un lado por la luz ultravioleta y por otro por la infrarroja.

La actividad solar misma, específicamente la radiación ultravioleta ha sido vinculada con mutaciones en los genes que llevarían a una mayor prevalencia de enfermedades por daño en el DNA. Esta acción de los rayos ultravioletas emitidos en diferente intensidad a lo largo del ciclo solar sería responsable de las alteraciones genéticas al largo plazo, vinculadas a enfermedades metabólicas, autoinmunes, trastornos mentales y neoplasias ${ }^{25,26}$. Sin embargo, no se ha correlacionado la actividad solar con la incidencia de trastornos anímicos (manías y depresiones) que ocurren en forma paralela con la actividad solar.

En una comunicación anterior, se correlacionó la actividad solar con la aparición de trastornos afectivos durante un ciclo solar completo (11 años), encontrándose que los desórdenes depresivos poseen una correlación inversa con la actividad solar, mientras que las manías se vincularon en forma directa con la energía proveniente del $\mathrm{Sol}^{27}$.

El presente trabajo intenta relacionar la actividad solar con la aparición de trastornos afectivos durante 16 años, casi un ciclo solar y medio.

\section{Material y Método}

Se revisaron 1.862 historias clínicas de sujetos que poseían diagnósticos de cuadros afectivos y que estuvieron hospitalizados en la Clínica Psiquiátrica Universitaria (CPU) de la Universidad de Chile, en Santiago, durante los años 1990 y 2005. El diagnóstico, tanto de los cuadros depresivos mayores y de bipolaridad, se ajustó a los propuestos por el DSM III-R y DSM IV, sistemas clasificatorios semejantes en los criterios diagnósticos de depresión mayor y manía ${ }^{28,29}$. Es decir, no se incorporaron depresiones en que el factor reactivo o adaptativo ameritaba esta categoría o distimias u otros cuadros de diversas etiologías, como las orgánicas. Para evaluar la relación con 
la actividad solar, sólo se consideró la incidencia de primeras hospitalizaciones de depresiones mayores y cuadros maníacos que cumplieran con los requisitos de no poseer hospitalizaciones ni tratamientos previos.

La incidencia de sujetos con depresión mayor y cuadros maníacos fue correlacionada con la actividad solar medida por el número de Wolf durante el mismo período anual, comprendido entre los años 1990 y 2005, ambos inclusive. Se calculó el coeficiente del rango de correlación de Spearman y la recta de regresión para aquellas variables que se asociaron significativamente. El nivel se significación fue fijado en 0,05.

El número de Wolf varía diariamente y está dado por la fórmula $R=K(10 g+f)$, donde " $K$ " es una constante que depende del observador, "g", el número de grupos de manchas solares y "f", el número total de manchas. Para los efectos del estudio, se consideró el número de Wolf promedio para los años mencionados.

\section{Resultados}

Los requisitos de inclusión fueron cumplidos por 450 historias clínicas, de las cuales 299 (66\%), presentaron cuadros depresivos (199 mujeres) y 151, 34\%, manías (73 mujeres) como diagnósticos de sus primeras hospitalizaciones. La edad de aparición de la depresión ocurrió entre los 15 y 72 años, con un promedio de 40 años. Para las manías fue entre 15 y 62 años, con un promedio de 28 años.
La Figura 1 muestra los índices de actividad solar medida por el número de Wolf; la Figura 2, la incidencia de hospitalizaciones de depresiones mayores y manías entre los años 1990 y 2005 en la CPU, Universidad de Chile.

Se observa que las depresiones aumentaron su incidencia de hospitalizaciones durante los años en que la actividad solar medida por el número de Wolf disminuyó, lo que corresponde a períodos de disminución de energía liberada por el sol. Por el contrario, existe una tendencia en las manías a disminuir su incidencia de hospitalizaciones durante los años en que la actividad solar disminuye y a aumentar su frecuencia en años de mayor actividad solar.

Al contrastar los datos de la Figura 1 (actividad solar) con los de la Figura 2 (incidencia de manías y depresiones), se aprecia que los cuadros depresivos mostraron una correlación estadísticamente significativa con la actividad solar, siendo ésta negativa, con un coeficiente de rango de correlación de Spearman de $-0,812(\mathrm{p}=0,000)$. En la Figura 3 se aprecia que las depresiones disminuyen su incidencia en la medida que aumenta la actividad solar medida por el número de Wolf. Los maníacos no mostraron una correlación con la actividad solar, con un coeficiente de rango de correlación de Spearman de 0,399 ( $\mathrm{p}=0,063)$, cercano a la significación estadística.

Se calculó la asociación entre el número de Wolf y las depresiones, las que quedan representadas por el modelo lineal mediante la fórmula: $n .^{\circ}$ de depresiones $=25,43$ - $\left(0,095 \times \mathrm{n}^{\circ}\right.$ de Wolf $)$, en

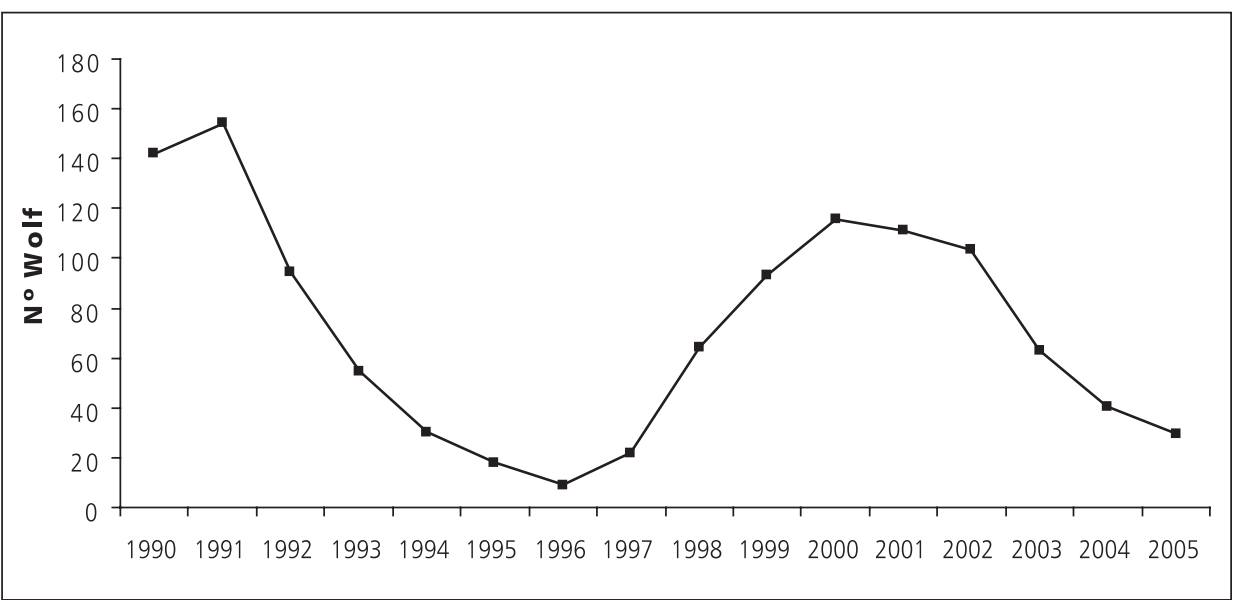

Figura 1. Número promedio de Wolf para los años 1990-2005. 


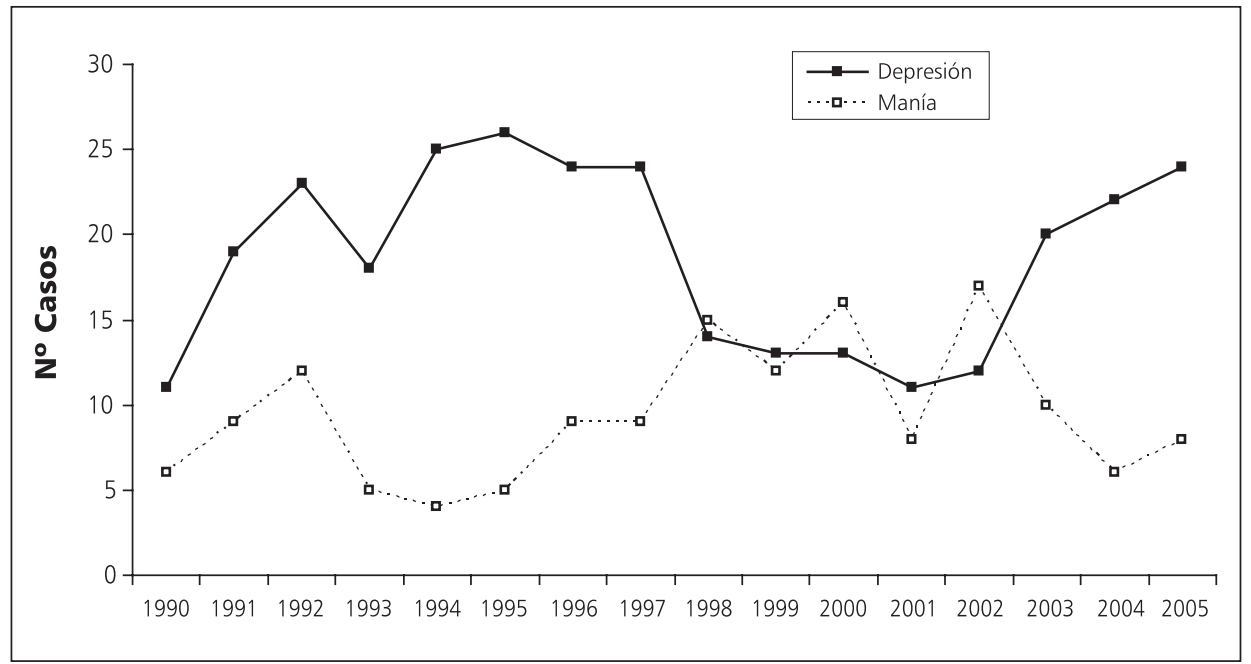

Figura 2. Incidencia de hospitalizaciones por depresión mayor y manía entre los años 1990-2005.

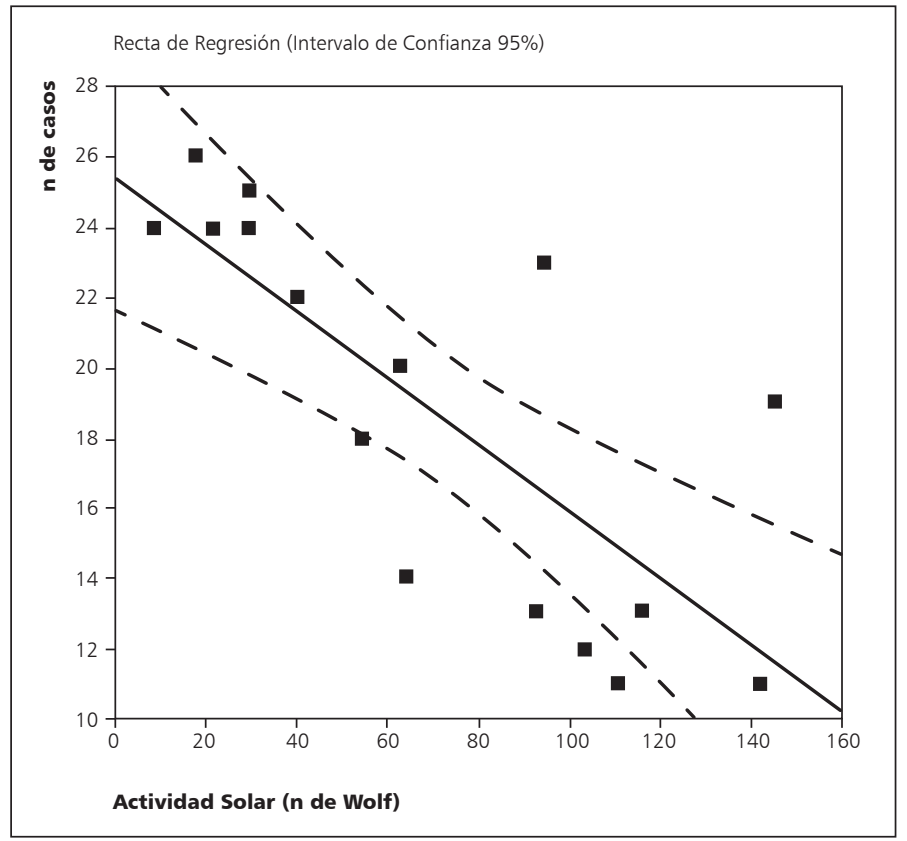

Figura 3. Correlación entre casos de depresión y actividad solar.

que la constante es de 25,43 y la pendiente 0,095 para explicar la variable dependiente $\left(n .^{\circ}\right.$ de depresiones) por la variable independiente (actividad solar expresada por el número de Wolf).

\section{Discusión}

Algunas consideraciones se pueden efectuar acerca de la actividad solar. El número de Wolf representa un índice de la energía liberada por el sol hacia la tierra. La actividad solar comprende no sólo la cantidad de luz visible que llega a la tierra en un momento dado, sino también la energía contenida en los rayos gama, rayos $\mathrm{x}$, luz ultravioleta, siendo éstos últimos los principales responsables de la energía solar, que en último término es lo que indica el número de Wolf. La luz visible sólo es una fracción ínfima del campo de las radiaciones electromagnéticas solares. El espectro visible de la luz está limitado por la zona infrarroja que posee una 
mayor longitud de onda y por la luz ultravioleta, seguida de los rayos $\mathrm{x}$ y rayos gama de aún menor longitud de onda ${ }^{30}$.

El comportamiento de las manchas solares no es uniforme sino que, por el contrario, su presencia y actividad varía diariamente, por meses y años, adquiriendo diversas dimensiones y que, en oportunidades, se pueden observar a simple vista en el disco solar.

Existe una clara relación entre las manchas medidas por el número de Wolf y la actividad magnética del sol. El magnetismo solar se aprecia en relación al auge y disminución de las manchas solares $^{24,30-33}$. La intensidad del campo magnético en una mancha solar se eleva a cifras entre 2.000 a 4.000 oersted, siendo la del campo terrestre 0,5 oersted. De este modo, variaciones en el número de las manchas implica también cambios de relevancia en la actividad magnética solar. La intensidad máxima del campo magnético corresponde al centro de la mancha, disminuyendo en forma progresiva hacia la periferia. Una mancha más oscura y más fría emitirá menos luz, pero presentará una intensidad magnética superior.

Las manchas solares están rodeadas a su vez por zonas brillantes denominadas fáculas, que son las principales responsables de la luz visible proveniente del sol, las que están separadas entre sí por una región de penumbra. Éstas son relieves que sobresalen de la superficie solar y que son más brillantes que la fotosfera, estando constituidas por enormes nubes luminosas que dan lugar a erupciones cromosféricas o fulgores. Estas fáculas poseen una zona central caliente y liberan energía en forma de luz, lo que se puede observar a simple vista particularmente en los bordes del sol, ya que la luminosidad de la fotosfera disminuye en dichas zonas. Luego de una intensa actividad, se reducen hasta desaparecer, por lo que en un intervalo de 11 años se encuentran tanto variaciones del área total de las manchas como de las fáculas ${ }^{24}$.

El aumento y disminución periódica de las manchas solares se acompaña de una consecuente variación de las fáculas, pues, en la medida que aumentan las manchas, aparecen zonas brillantes en los bordes de éstas, por lo que la disminución de la cantidad de luz proveniente de las manchas se vincula simultáneamente con una mayor cantidad de luz proveniente de las fáculas.

El presente trabajo estudió la actividad solar medida por el número de Wolf, que señala la can- tidad de manchas, lo que incluye también, pero en forma indirecta, la presencia de fáculas que emiten energía luminosa, cuya aparición sigue un patrón semejante a las manchas solares.

Sin embargo, algunas consideraciones se deben efectuar acerca de las variables que intervienen. En el presente trabajo, el cálculo del número de hospitalizaciones se efectuó sin considerar el recuento de hospitalizaciones de sujetos portadores de otras patologías, por lo que se trata de una incidencia anual de únicamente los cuadros mencionados. Tampoco se ha considerado la incidencia de sujetos con manías o depresiones con antecedentes de hospitalizaciones previas o consultas ambulatorias, debido al sesgo que provoca el uso de fármacos o psicoterapia a que hayan estado expuestos estos sujetos. La población que atiende la Clínica Psiquiátrica Universitaria de la Universidad de Chile no corresponde sólo a un sector de la ciudad con parámetros definidos desde el punto de vista sociodemográfico, aunque la población atendida tiende a ser estable durante los años incluidos en el estudio.

Los resultados aquí encontrados pueden representar un inicio en el estudio de la variable "actividad solar" sobre los cuadros anímicos, Hasta la fecha se ha estudiado la relación de la actividad solar, especialmente la radiación ultravioleta, con posibles mutaciones genéticas que antecederían a variados cuadros clínicos como enfermedades autoinmunes y otras ${ }^{25,26}$. En este trabajo, como se ha planteado, se ha vinculado la actividad solar con la incidencia de los trastornos anímicos, estableciendo un paralelismo entre ambas variables.

Por otro lado, en un informe previo ${ }^{27}$ se estableció la relación entre la actividad solar y los trastornos del ánimo en un solo ciclo solar de 11 años. El presente estudio considera un período más prolongado de tiempo, de 16 años (un ciclo solar y medio aproximadamente) que muestra resultados semejantes a este estudio previo, es decir, que persiste la relación entre menor actividad solar y aumento del número de depresiones, estadísticamente significativa junto a un relativo aumento de las manías, no estadísticamente significativa con respecto a los años de mayor actividad solar.

Conclusiones más definitivas podrían lograrse en la medida que los hallazgos aquí expuestos se observen en otros lugares de la tierra, en latitudes diferentes o en distintos continentes del globo terráqueo. 


\section{Conclusiones}

Los cuadros depresivos mostraron un comportamiento diferente a las manías. En los primeros, existe una relación inversa, estadísticamente significativa con la actividad solar, mientras que las manías muestran una correspondencia positiva con la actividad solar, no estadísticamente significativa. Se observó una mayor incidencia de hospitalizaciones de depresiones en años con menor actividad solar, con una leve tendencia de los maníacos para hospitalizarse en años con mayor actividad solar.

Los estudios sobre la actividad solar indican que la energía total irradiada por el sol, incluyendo a todo el espectro electromagnético, varía entre varios centenares y millares por ciento, mostrando una periodicidad relacionada con el número de manchas solares, energía que puede modificar algunos parámetros biológicos como los expuestos en el presente estudio.

La presencia y actividad de las manchas solares están sometidas en la actualidad a una constante revisión, por lo que éstas u otras hipótesis deberían ser dilucidadas en posteriores estudios para comprender los hallazgos aquí presentados, que señalan una incidencia mayor de depresiones en años de menor actividad solar, junto a una mayor incidencia de manías en años de mayor actividad solar.

Agradecimientos: Agradecemos la colaboración de la Dra. Rosemarie Fritsch, quien prestó la asesoría estadística al presente estudio.

\section{Referencias}

1. Magnusson A. An overview of epidemiological studies on seasonal affective disorder. Acta Psychiatr Scand 2000; 101: 176-84.

2. Wehr TA, Sack DA, Rosenthal NE. Seasonal affective disorder with summer depression and winter hypomania. Am J Psychiatry 1987; 144: 1602-3.

3. Potkin SG, Zetin M, Stamenkovic V, Kripke D, Bunney WJ. Seasonal affective disorder: prevalence varies with latitude and climate. Clin Neuropharmacol 1986; 4: 181-3.

4. Rosen LN, Targum SD, Terman W, Bryantt MJ, Hoffman $\mathrm{H}$, Kasper SF, et al. Prevalence of seasonal affective disorder at four latitudes. Psychiatry Res 1990; 31: 131-44.

5. Benedetti F, Colombo C, Barbini B, Campori E, Smeraldi
E. Morning sunlight reduces length of hospitalization in bipolar depression. J Affect Dis 2001; 62: 221-3.

6. Molin J, Mellerup E, Bolwig T, Scheike T, Dam H. The influence of climate on development of winter depression. J Affect Dis 1996; 37: 151-5.

7. Young MA, Meaden PM, Fogg LF, Cherin EA, Eatman CI. Which environmental variables are related to the onset of seasonal affective disorder? J Abnorm Psychol 1997; 106: 554-62.

8. Metzger J, Berthou V, Perrin P, Sichel J. Phototherapy: Clinical and therapeutic results of two years experience. Encephale 1998; 24: 480-5.

9. Lee T, Blashko C, Jansen H, Paterson J, Chan C. Pathophysiological mechanism of seasonal affective disorder. J Affect Dis 1997; 46: 25-38.

10. Reme CE, Grimm C, Hafezi F, Wenzel A. Lamp standars and ocular safety. En: Partonen T, Magnusson A, ed. Seasonal Affective Disorder. Practice and research. New York : Oxford University Press, 2001; 79-84

11. Lewy AJ, Wehr TA, Goodwin FK, Newsome DA, Markey SP. Light suppresses melatonin secretion in humans. Science 1980; 210: 1267-9.

12. Rosenthal NE, Sacda, Gillin JC, Lewy AJ, Goodwin FK, Davenport, et al. Seasonal affective disorder. A description of the syndrome and preliminary findings with light therapy. Arch Gen Psychiatr 1984; 41: 72-80.

13. Gaddy JR, Rollag MD, Ruberg FL, Brainard GC. Lightinduced melatonin suppression and pupil size. Sleep research 1993; 22: 406.

14. Czeisler CA, Kronauer RE, Allan JS, Duffy JF, Jewett ME, Brown EN, et al. Bright light induction of strong (Type 0 ) resetting of the human circadian pacemaker. Science 1989; 244: 1328-33.

15. Minors DS, Waterhouse JM, Wirz-Justice A. A human phase-response curve to light. Neurosci Lett 1991; 133: 36-40.

16. Boivin DB, Duffy JF, Kronauer RE, Czeisler CA. Doseresponse relationships for resetting of human circadian clock by light. Nature 1996; 379: 540-2.

17. Boivin DB, Czeisler CA. Resetting of circadian melatonin and cortisol rhythms in humans by ordinary room light. Neuroreport 1998; 9: 779-82.

18. Waterhouse J, Minors D, Folkard S, Owens D, Atkinsons G, Macdonald T, et al. Light of domestic intensity produces phase shifts of the circadian oscillator in humans. Neurosci Lett 1998; 245: 97-100.

19. Wehr TA, Giesen HA, Moul DE, Turner EH, Schwartz PJ. Supresion of men's responses to seasonal changes in day length by modern artificial lighting. Am J Psysiol 1995; 269: $173-8$.

20. Matthews CD, Guerin MV, Wang X. Human plasma 
melatonin and urinary 6-sulphatoxy melatonin: studies in natural annual photoperiod abd in extended darkness. Clin Endocrinol 1991; 35: 21-7.

21. Werh TA, Moul DE, Barbato G, Giesen HA, Seidel JA, Barker C, et al. Conservation of photoperiod-responsive mechanism in humans. Am J Psysiol 1993; 265: R846-57.

22. Wehr TA. The durations of human melatonin secretion and sleep respond to changes in daylenght (photoperiod). J Clin Endocrinol Metab 1991; 73: 1276-80.

23. Beullens J. Melatonin and affective disorders. Acta Neuropsychiat 1995; 7: 75-9.

24. Abetti G. El sol. Buenos Aires: Eudeba; 1962; 67-170.

25. Davis Ge, Lowell W. Solar cycles and their relationship to human disease and adaptability. Medical Hypotheses 2006; 67: 447-61.

26. Davis GE, Lowell W. Chaotic solar cycles modúlate the incidence and severity of mental illness. Medical Hy- potheses 2004; 62: 207-14.

27. Ivanovic-Zuvic F, De La Vega R, Ivanovic-Zuvic N, Renteria P. Enfermedades afectivas y actividad solar. Actas Esp Psiquiatr 2005; 33: 7-12.

28. American Psychiatric Association. DSM-III-R. Manual diagnóstico y estadístico de los trastornos mentales. Barcelona: Masson SA, 1988.

29. American Psychiatric Association. DSM-IV. Manual diagnóstico y estadístico de los trastornos mentales. Barcelona: Masson SA, 1995.

30. Rousseau P. La luz. Buenos Aires: El Ateneo; 1978; 17-34.

31. Parker EM. El sol. Selecciones de Scientific American. Madrid: H Blume Ediciones; 1977; 35-43.

32. Rudaux L, Vaucouleurs G. Astronomía. Barcelona: Labor; 1962; 330-70.

33. Mesme-Rides E, Baliunas S, Sokoloff D. The stellar dinamo. Scient Am 1996; 8: 45-52. 